\title{
Development and Testing of an Automatic Turning Movement Identification System at Signalized Intersections
}

\author{
Kun $\mathrm{Xu}^{1,2}$, Ping $\mathrm{Yi}^{2,3^{*}}$, Chun Shao ${ }^{3}$, Jialei $\mathrm{Mao}^{3}$ \\ ${ }^{1}$ State Key Laboratory of Information Engineering in Surveying, Mapping and Remote Sensing, Wuhan University, Wuhan, China \\ ${ }^{2}$ Intelligent Transportation System Research Center, Wuhan University of Technology, Wuhan, China \\ ${ }^{3}$ Department of Civil Engineering, the University of Akron, Akron, USA \\ Email: xk_ben@126.com, ${ }^{*}$ pyi@uakron.edu
}

Received May 31, 2013; revised July 2, 2013; accepted August 5, 2013

Copyright (C) $2013 \mathrm{Kun} \mathrm{Xu}$ et al. This is an open access article distributed under the Creative Commons Attribution License, which permits unrestricted use, distribution, and reproduction in any medium, provided the original work is properly cited.

\begin{abstract}
Vehicle turning movement data from signalized intersections is utilized for numerous applications in the field of transportation. Such applications include real-time adaptive signal control, dynamic traffic assignment, and traffic demand estimation. However, it is very time consuming and costly to obtain vehicle turning movement information manually. Previous efforts to simplify this process were focused on solving the problem using an O-D matrix, but this method proved to be inaccurate and unreliable with the existing data acquisition system. Another study involved the identification of vehicle turning movements from the detector information, but the presence of shared lanes led to uncertainties in vehicle matching, thus limiting application of the method only to intersections without shared lanes. In light of those unsuccessful attempts, this paper develops and tests a system called the Automatic Turning Movement Identification System (ATMIS), which estimates vehicle turning movements at a signalized intersection in real time, regardless of its geometry. The results from lab experiments as well as a field test show that the algorithm is very promising and may potentially be expanded for field applications.
\end{abstract}

Keywords: Turning Movement Identification; Signalized Intersection; Detector and Detection; Vehicle Matching; Lab Experiment and Field Test

\section{Introduction}

Turning Movement Information (TMI) relates to the number of vehicles completing left or right turns or through movements from each intersection approach. This information is utilized in many transportation applications and is vital for the improvement of intersection operations. In addition to routine traffic applications, the information can be applied to dynamic traffic assignment, adaptive signal control, and transportation planning. Therefore, it is important to obtain such information accurately and timely in a cost-effective manner.

The traditional method for gathering TMI is a very lengthy process in which observers manually collect data pertaining to the turning movements at a particular intersection. This method of data collection is not practical in real time and therefore cannot be used for such applications as adaptive signal control logic [1]. With the improvement in modern vehicle detection systems, Cremer and Keller sought to automatically handle this process by using the O-D matrix method as first introduced in 1981

\footnotetext{
"Corresponding author.
}

[2]. Later, Nihan and Davis [3] as well as Cremer and Keller [4] developed a set of dynamic O-D estimation models for intersections and small networks based on the prediction-error minimization method. In their models, the volumes of vehicles flowing in and out of each intersection approach are used as input parameters. Given these parameters, the O-D matrix is then solved to get the turning movement information. Due to limited volume data available and the fluctuations of traffic flow, the results obtained by the O-D matrix method are not accurate for potential applications. In addition, the data collection time interval used is too long for this method to be suitable for gathering real-time TMI. Yet another method was developed by Jiao et al. [5] using the Genetic Algorithm to identify TMI. This approach was first introduced in 2003, and the simulation results were encouraging. However, this method is very sensitive to detection errors commonly seen in the field, associated with the type of detectors used, data reporting intervals, weather conditions, and the geometry of the intersection.

Virkler and Kumar [6,7] at the University of Missouri-Columbia developed and tested the Time and Place 
Systems (TAPS) method utilizing the phasing and detection information for TMI. TAPS separates right turning vehicles from the through traffic flow using an exclusive lane detector but the left turning vehicles in a shared lane can cause the error to vary from $5 \%$ to $70 \%$. In recent years, Chen et al. [8] used a path flow estimator to derive TMI for an entire roadway network and Zhang et al. [9] tested a nonlinear programming approach to calculate intersection O-D matrix. Those methods rely on accurate data from a large number of detectors to ensure feasible and stable solutions to the mathematical models; in addition, the TMI is not obtained in small time intervals in support of real time applications.

To make further improvements on the above subject this paper develops and tests a system called the Automatic Turning Movement Identification System (ATMIS). In particular, this system aims at improving data accuracy at intersections and the algorithm is designed to treat shared lanes in different lane configurations.

\section{ATMIS Algorithm}

The algorithm used in ATMIS makes use of information from both the detectors and the signal system of the intersection. By tracking each detector's status and traffic signal operation by the second, vehicle turning movements can be calculated. To illustrate the algorithm, consider a four-legged intersection as shown in Figure 1. In this configuration, there is only one lane at each intersection leg, which means that each lane is shared by left turning, right turning, and through vehicles alike. To determine the vehicle turning movements automatically, data obtained from the detectors originally deployed in the intersection for signal control will be used. According to its function, there are two types of detectors: input detectors (white) provide the detection of vehicles approaching the intersection while output detectors (gray) provide the detection of vehicles leaving the intersection.

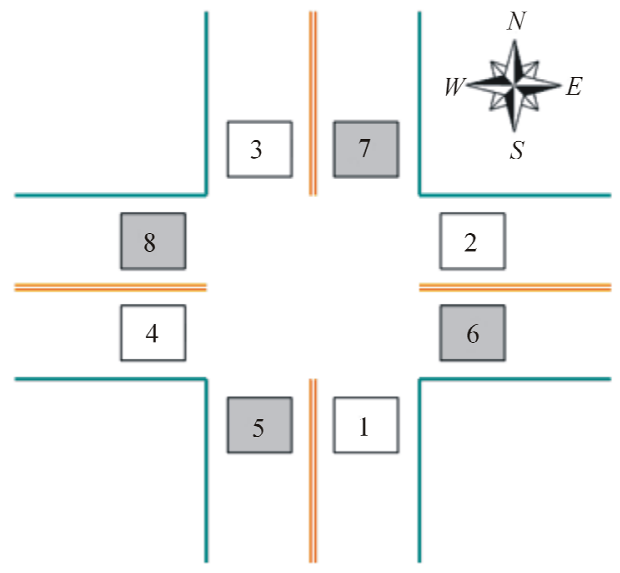

Figure 1. Detector locations at typical four-legged intersecttion.
For each turning movement, there is a fixed detector pair to be activated. For example, any northbound left-turning vehicle will first activate detector 1 and then detector 8 . Similarly, every turning movement throughout the intersection can be defined by a sequence of detections, and the resulting sequences are shown in Table 1. The algorithm is used to determine vehicle turning movements from a specific detection sequence according to the records of data collected by each detector.

At a signalized intersection, only a fraction of the approaching vehicles can be given the right of way at one moment. Knowledge of the signal phases can help narrow down the candidate movements. However, the process of identifying turning movements from a detector pair can still be very complicated when there are multiple possibilities caused by the presence of a shared lane. For instance, a vehicle from detector 6 can be paired with that from detector 1 to provide a northbound right turn, or it can be paired with detector 3 to yield a southbound left turn. The purpose of the algorithm is to identify the turning movement under such complicated circumstances, independent of the geometry of the intersection. The algorithm is supported by three modules to handle all possible conditions: the Input Detection Recording Module, the Output Detection Matching Module, and the Input Detection Cleanup Module. Figure 2 introduces the detailed procedures of these three modules.

\subsection{Input Detection Recording Module}

This module is triggered by data received from input detectors, represented by detectors 1 to 4 in Figure 1. Once the status of an input detector changes from activated to deactivated, signifying that the vehicle no longer

Table 1. Turning movement table.

\begin{tabular}{cccc}
\hline Input Det. & Output Det. & Movement & \\
\hline 1 & 7 & Northbound Through & NT \\
1 & 6 & Northbound Right Turn & NR \\
1 & 8 & Northbound Left Turn & NL \\
2 & 8 & Westbound Through & WT \\
2 & 7 & Westbound Right Turn & WR \\
2 & 5 & Westbound Left Turn & WL \\
3 & 5 & Southbound Through & ST \\
3 & 8 & Southbound Right Turn & SR \\
3 & 6 & Southbound Left Turn & SL \\
4 & 6 & Eastbound Through & ET \\
4 & 5 & Eastbound Right Turn & ER \\
4 & 7 & Eastbound Left Turn & EL \\
\hline
\end{tabular}




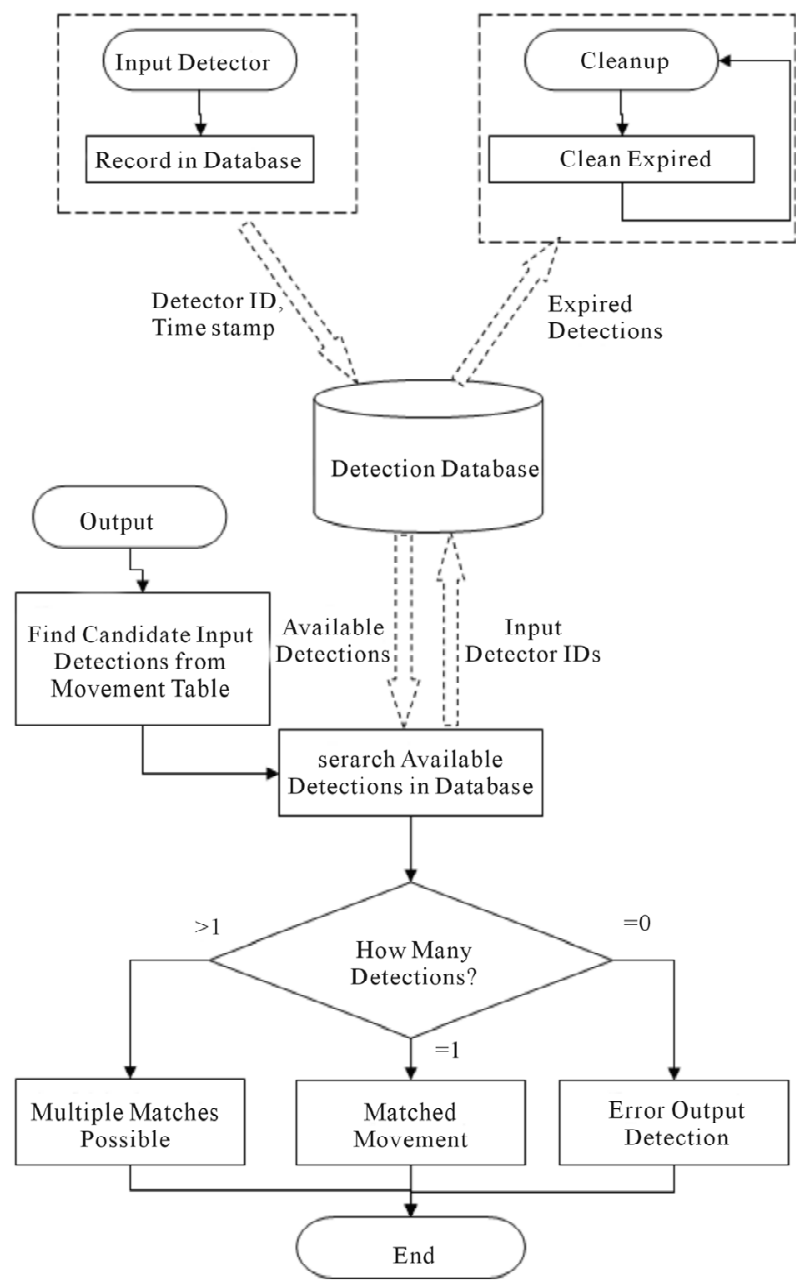

Figure 2. Flowchart of ATMIS algorithm.

occupies the detector, the detector's ID and the deactivation timestamp are recorded and sent to the database. The timestamp is used for two purposes; the first is to match the sequence of the detector pair, and the second is to time-out unmatched detections. The input detections are to be used later in the other two modules.

\subsection{Output Detection Matching Module}

This module is triggered by data received from output detectors, or detectors 5 to 8 in Figure 1. An output detection is reported when a vehicle has left the detector, and there should be one and only one matching input detection to define the movement as completed. In practice, however, the algorithm will not always return a single matched input detection from the database. As shown in Figure 2, there exist three possible conditions, as described below:

1) There is no matched detection in the database.

An unmatched detection can be caused either by misdetection from input detectors or false detection from output detectors. Based on the timestamp associated with the output data, the algorithm determines that there is no possible input match for the detection. No future detection will provide a match for the error, and the output detection is simply ignored in the algorithm. The error is then recorded and reported by the system.

2) Only one detection match in the database.

This is the best condition for a detection match. The times at which the detections were received allow the system to return a single possible detector pair. The system simply searches the turning movement table to locate the corresponding movement for the given detection pair. The corresponding input and output detections are then removed from the unmatched list in the database.

3) Multiple detection matches in the database.

This is the most complicated situation to be addressed by the algorithm. The basic idea behind the process can be illustrated using a simple example. Once again, consider a four-legged intersection with one lane in each direction as shown in Figure 3. There is one vehicle moving from south to east (northbound right turn) while another vehicle travels from north to south (southbound through). At one moment, the vehicles' positions are shown in Figure 3(a), and two input detections from detectors 1 and 3 are obtained. When a vehicle leaves detector 6, one output detection will be sent to the system to locate the possible input detection from the database. At this point, the system cannot tell which combination (either with detector 1 or 3 ) is correct when the signal indication is green for the north-south direction, so the algorithm will hold all the detection data and do not output anything.

The multiple matches can be broken up when both vehicles have left the intersection. As shown in Figure 3(b), two output detections are now recorded in the database, including one from detector 5 . Since a vehicle movement from detector 1 to detector 5 would effectively mean a U-turn, which is not allowed in most cases, only one input detection from detector 3 can be a feasible match for output detection 5 at this time. Thus, the algorithm will output the movement "southbound through" and "northbound right turn" and clear the above recorded detections from the database. In practice, many complex situations like this exist and the algorithm is designed to handle all the possibilities.

\subsection{Input Detection Cleanup Module}

This module is an independent process to clean up the expired input detection data. Input detections that have no matched output detections over a given time interval should be removed from the database to help control the accumulation of multiple unmatched detections. The unmatched detections may be caused by false detection from an input detector or misdetection on an output detector. The cleanup is done at every second and the expi- 
ration time period is determined according to the specific turning movement trajectory, the length of each relevant signal phase, and the current signal status.

\section{Experiments and Results}

The algorithm of ATMIS has been implemented with the Visual Studio software. To test the feasibility of the algorithm, lab experiments and a field test were conducted in a NEMA standard control cabinet with an Autoscope [10] visual detection system. The difference between lab experiments and field test is the video source from the detection system. In lab experiments, the detection system uses recorded video as the input while in the field test a live feed is utilized. Detection and traffic signal information is collected by a personal computer with an interface card installed in the signal cabinet. The estimated turning movements are shown on the computer screen in real time.

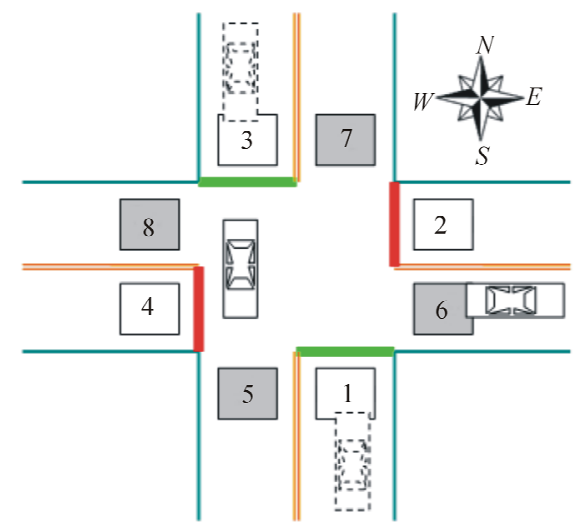

Input Detections: 1, 3; Output Detection: 6 Possible Matches: $1 \rightarrow 6$, Northbound Right Turn

$3 \rightarrow 6$, Southbound Left Turn

(a)

\subsection{Lab Experiments}

Two intersections have been selected in lab experiments, including the intersections of Fifth Avenue and South Arlington Street, and East Wilbeth Road and South Arlington Street in Akron, Ohio. These two intersections are all video camera equipped, and their geometries are quite different. As shown in Figure 4(a), the intersection of Fifth Avenue and South Arlington Street is a standard four-legged intersection with shared lanes. The intersecttion of East Wilbeth Road and South Arlington Street, shown in Figure 4(b), is a T-intersection with only one lane shared by right turns and through traffic.

For each intersection, daytime traffic was captured by video footage, and the duration of each video varied from 60 minutes to 100 minutes. The ground truth of the turning movements was also collected manually in 20-minute intervals. The results of the experiments at the two intersections are depicted in Figures 5 and 6. Those figures

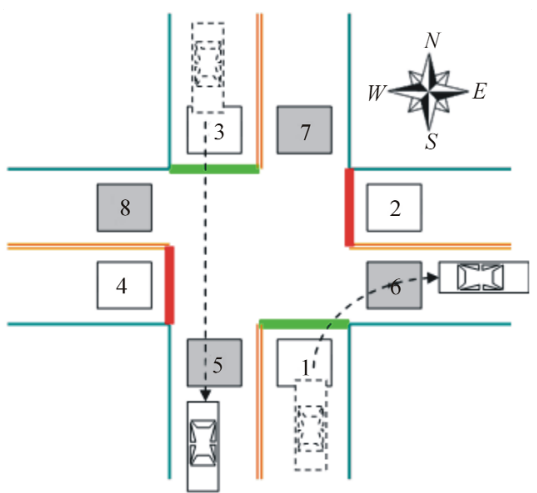

Input Detections: 1, 3; Output Detection: 5, 6

Possible Matches: $1 \rightarrow 6$, Northbound Right Turn

$3 \rightarrow 6$, Southbound Left Turn

$3 \rightarrow 5$, Southbound Through

(b)

Figure 3. Input and output detections matching. (a) Undetermined detection matching; (b) Detection matched after both vehicles cleared the intersection.

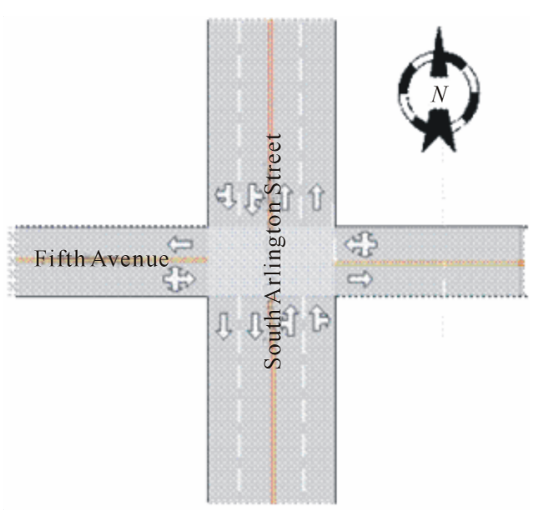

(a)

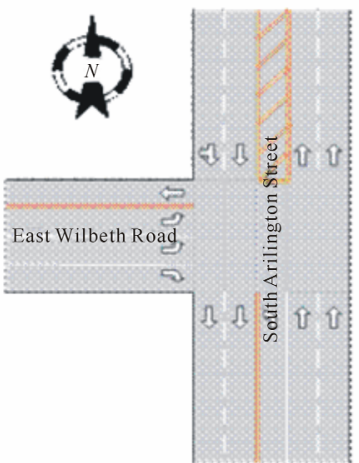

(b)

Figure 4. Intersections used in lab experiments. (a) Fifth Ave. and South Arlington Street; (b) East Wilbeth Rd. and South Arlington Street. 


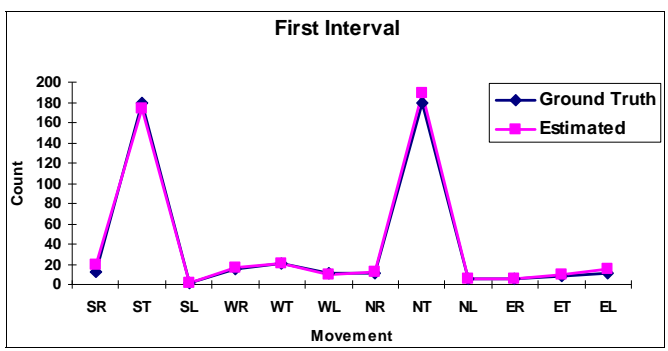

(a)

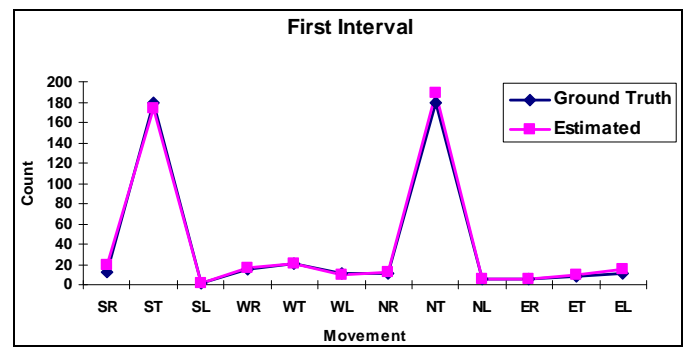

(c)

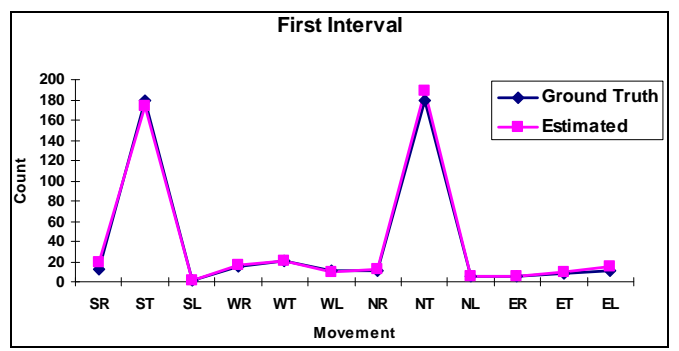

(b)

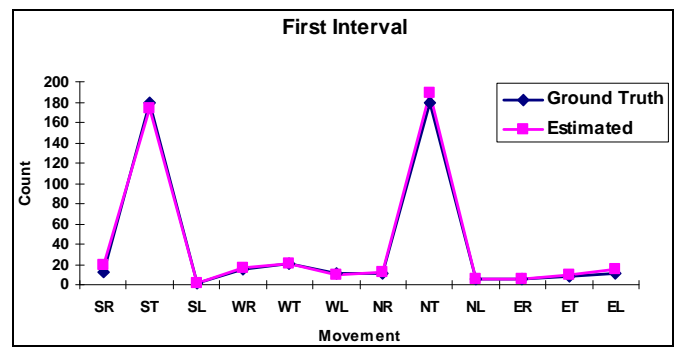

(d)

Figure 5. Lab experiment results, Fifth Avenue and South Arlington Street.

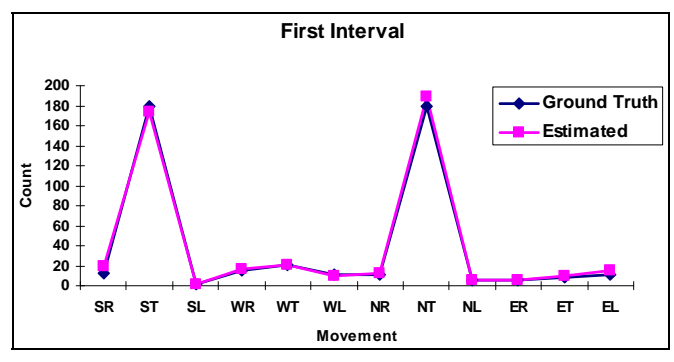

(a)

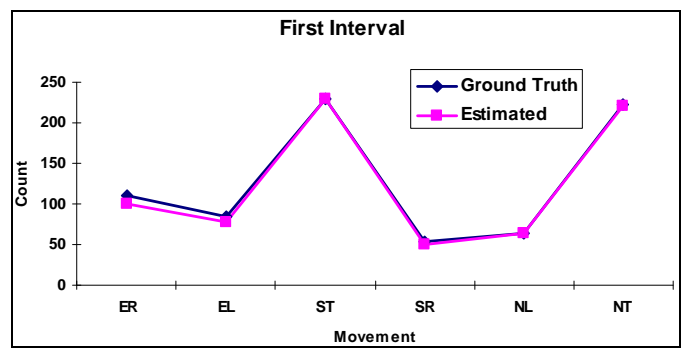

(b)

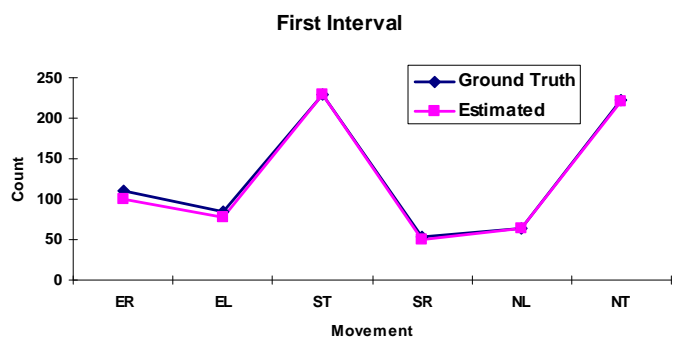

(d)

Figure 6. Lab experiment results, East Wilbeth Road and South Arlington Street.

show that the estimated vehicle turning movements resulting from ATMIS match very closely to the ground truth data. As shown in Table 2, the error percentages in both cases are relatively small and so are the differences between the percentages between the intersections $(4.94 \%$ vs. 5.30\%). This indicates that ATMIS works well regardless of the number of shared lanes included in the two intersections.

It should be noted that the detection error (missing or double counts by the video system) was around $20 \%$ during each experiment. This further demonstrates that ATMIS has good error correction ability.

\subsection{Field Test}

Although lab experiments were very encouraging, a field test was further performed to verify the accuracy of the ATMIS system in practical applications. This field test took place on a normal working Monday from 11:30 AM to $12: 10 \mathrm{PM}$ at the intersection of Fifth Avenue and South Arlington Road and the test results are shown in Figure 7. Compared with the ground truth, the average error was $7.89 \%$, which is slightly higher than that in the prior lab experiment. Further investigation into the detection data revealed that during the field test the video de- 
Table 2. Matched turning movements and error percentage of lab experiments.

\begin{tabular}{ccccc}
\hline \multirow{2}{*}{ Time Interval } & \multicolumn{2}{c}{ Fifth \& Arlington } & \multicolumn{2}{c}{ Wilbeth \& Arlington } \\
\cline { 2 - 5 } & Matched Pairs & Error \% & Matched Pairs & Error \% \\
\hline 1 & 461 & $6.49 \%$ & 764 & $3.14 \%$ \\
2 & 496 & $5.44 \%$ & 811 & $6.66 \%$ \\
3 & 505 & $4.75 \%$ & 804 & $5.97 \%$ \\
4 & 518 & $3.28 \%$ & No data & N/A \\
Average & 495 & $4.94 \%$ & 793 & $5.30 \%$ \\
\hline
\end{tabular}

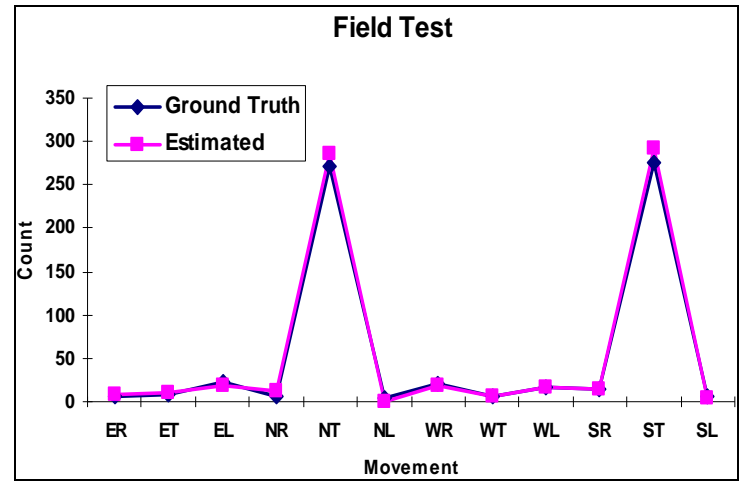

Figure 7. Field test results on Fifth Avenue and South Arlington Street.

tection system had an error rate from $25 \%$ to $30 \%$ on that day. Due to site restrictions, the authors were not able to go back to and repeat the field test. Nevertheless, the results obtained from the test have demonstrated the data effectiveness and preliminary feasibility of ATMIS for field applications. When more time is given to make adjustments to the detector positions and calibrate the video system parameters in the future, the results are expected to further improve.

\section{Conclusion}

Based on the experiments, the results provided by ATMIS are quite encouraging with an average error of $5 \%$ in lab experiments and $8 \%$ in preliminary field test. The reason for the estimation errors have been discussed, and improvements are expected when the data errors due to the video detection system are reduced. Although the testing effort is limited the results have shown that ATMIS is very promising with small estimation errors and the algorithm is not noticeably affected by the presence of shared lanes. Further work will be conducted in the related areas to expand the scope of applications and improve the system performance.

\section{REFERENCES}

[1] Transportation Research Board, "Highway Capacity Man- ual," Washington DC, 2000.

[2] M. Cremer and H. Keller, "Dynamic Identification of O-D Flows from Traffic Counts at Complex Intersections," Proceedings of the 8th International Symposium on Transportation and Traffic Theory, Toronto, 22-24 June 1981, pp. 121-142.

[3] N. L. Nihan and G. A. Davis, "Recursive Estimation of Origin-destination Matrices from Input/Output Counts," Transportation Research B, Vol. 21, No. 2, 1987, pp. 149163. http://dx.doi.org/10.1016/0191-2615(87)90013-0

[4] M. Cremer and H. Keller, "A New Class of Dynamic Methods for the Identification of Origin-destination Flows," Transportation Research B, Vol. 21, No. 2, 1987, pp. 117132. http://dx.doi.org/10.1016/0191-2615(87)90011-7

[5] P. Jiao, H. Lu and L. Yang, "Real-Time Estimation of Turning Movement Proportions Based on Genetic Algorithm," Proceedings of the Conference on Intelligent Transportation Systems, Vienna, 13-15 September 2005, pp. 96-101.

[6] M. R. Virkler and N. Kumar, "System to Identify Turning Movements at Signalized Intersections," Journal of Transportation Engineering, Vol. 124, No. 6, 1998, pp. 607-609. http://dx.doi.org/10.1061/(ASCE)0733-947X(1998)124:6 (607)

[7] J. Tian, M. R. Virkler and C. Sun, "Field Testing for Automated Identification of Turning Movements at Signalized Intersection," Transportation Research Record: Journal of the Transportation Research Board, Vol. 1867, 2004, pp. 210-216. http://dx.doi.org/10.3141/1867-24

[8] A. Chen, P. Chootinan, S. Ryu, M. Lee and W. Recker, "An Intersection Turning Movement Estimation Procedure Based on Path Flow Estimator," Journal of Advanced Transportation, Vol. 46, No. 2, 2012, pp. 161-176. http://dx.doi.org/10.1002/atr.151

[9] X. Zhang, X. Cao and L. Sun, "Estimation of Intersection Turning Movement Proportion Using MATLAB," Proceedings of CICTP 2012, Beijing, 2012, pp. 822-828.

[10] P. B. Michalopoulos, B. Wolf and R. Benke, "Testing and Field Implementation of the Minnesota Video Detection System," Proceedings of 1990 Annual Meeting of the Transportation Research Board, Washington DC, 1990, pp. 176-184. 